\title{
Assessment of the Impact of a Sports Kit on Physical Activity in Children 8 to 11 Years of Age
}

\author{
Suzanne Lanckriet ${ }^{1}$, Elisabeth Brissieux ${ }^{1}$, Jean-Michel Borys ${ }^{2}$, Arnaud Jaruga ${ }^{1}$, Coralie Schnebelen-Berthier ${ }^{1}$, \\ Elise Dekneudt ${ }^{1}$, Alexandra Calais ${ }^{1}$, Pierre Richard ${ }^{2}$, Julie Mayer ${ }^{2}$, Caroline Bournez ${ }^{3}$, Julie Penin ${ }^{3}$, \\ Amar Djouak $^{4}$, Angela Chieh ${ }^{5}$, Alexis Normand ${ }^{5}$, Martine Duclos ${ }^{6,7,8}$, Jean-Michel Lecerf ${ }^{1, *}$ \\ ${ }^{1}$ Service de Nutrition, Institut Pasteur de Lille, Lille Cedex, France \\ ${ }^{2}$ EPODE INTERNATIONAL NETWORK, Paris, France \\ ${ }^{3}$ Communauté de communes Flandres Lys, La Gorgue, France \\ ${ }^{4}$ ISA Lille, - Groupe HEI+ISA+ISEN, Lille Cedex, France \\ ${ }^{5}$ Withings, Paris, France \\ ${ }^{6}$ Department of Sport Medicine and Functional Explorations, University-Hospital (CHU), G. Montpied Hospital, \\ Clermont-Ferrand, France \\ ${ }^{7}$ INRA, UMR 1019, UNH, CRNH Auvergne, CLERMONT-FERRAND, France \\ ${ }^{8}$ Clermont University, University of Auvergne, Unité de Nutrition Humaine, CLERMONT-FERRAND, France \\ *Corresponding author: jean-michel.lecerf@pasteur-lille.fr
}

\begin{abstract}
Objective: Fewer than 10\% of French children meet the WHO recommendations for physical activity (PA). As engaging in play activities is a key way to promote PA in children, we evaluated the effect of a sports kit called "Le Sport Ça Me Dit" ("Sport is fun") that provides a recreational way to engage in sports. Design: This was an open longitudinal controlled study. The children were divided into two groups for the first 7 weeks (Period 1): an Action group that used the kit for two recreational sessions per week (comprising six different activities) and a Control group that was not provided use of the kit. For the next 7 weeks (Period 2), neither group was provided use of the kit. Setting: Six towns in northern France. Subjects: 213 children aged 8-11 were recruited: 122 for the Control group and 91 for the Action group. Each child received a 3D accelerometer wristband to measure their level of PA during the 14 weeks of the study. In parallel, the children completed a questionnaire, 3 times (before (T0), during (T1) and at the end of the study (T2)), regarding their PA habits, screen time, and sleep habits. Results: A significant increase in the steps number was observed when the sports kit was used $(\mathrm{p}=0.025)$; although this effect was lost over time $(\mathrm{p}=0.672)$. Subpopulation analysis revealed a significant increase in the steps number and light PA in overweight children in the Action group for both periods $(\mathrm{p}=0.018$ and $\mathrm{p}=0.012$ ). Conclusion: The use of the sports kit increased the PA level of the children, and particularly in overweight children. Although these results require further investigation, they provide novel insights in regard to overweight children.
\end{abstract}

Keywords: physical activity, children, accelerometer, inactivity, overweight, QAPE

Cite This Article: Suzanne Lanckriet, Elisabeth Brissieux, Jean-Michel Borys, Arnaud Jaruga, Coralie Schnebelen-Berthier, Elise Dekneudt, Alexandra Calais, Pierre Richard, Julie Mayer, Caroline Bournez, Julie Penin, Amar Djouak, Angela Chieh, Alexis Normand, Martine Duclos, and Jean-Michel Lecerf, "Assessment of the Impact of a Sports Kit on Physical Activity in Children 8 to 11 Years of Age.” Journal of Physical Activity Research, vol. 2, no. 1 (2017): 50-60. doi: 10.12691/jpar-2-1-9.

\section{Introduction}

The health protective and health enhancing effects of physical activity (PA) on youths have been well documented $[1,2]$. There is an inverse relationships between the total physical activity and/or the total time accumulated in moderate-to-vigorous activity and the body's fat content [3], clustering of cardiovascular diseases, and metabolic risk factors [4,5]. Conversely, there is a positive relationships between PA and aerobic fitness in children [3]. Furthermore, excessive sedentary behaviors are associated with pediatric obesity and metabolic risks [6]. Recent studies have also shown that interrupting sedentary time with brief moderate-intensity walking can improve short-term metabolic function in children who are not overweight [7].

To maximize health benefits, WHO is recommended that children engage in $\geq 60$-minute of moderate-to-vigorous PA (MVPA) on a daily basis, while limiting their sedentary activities.

When assessing the level of daily PA in free-living conditions in children, accelerometers are considered to be one of the most effective ways to obtain objective information in regard to total PA, as well as the intensity, duration, and frequency of the PA performed [8]. Apete et al. [9] examined the compliance of children in France with PA guidelines. Based on objective assessments with accelerometers, they found that the proportion of children 
meeting the PA recommendations of $\geq 60 \mathrm{~min}^{-1} \mathrm{~d}^{-1}$ was between 5\% and 9\%. Furthermore, although children with normal weights spent up to $35 \%$ more time, on average, engaged in MVPA compared to overweight/obese children, a similar proportion of children in each weight category was sufficiently active according to the recommendations.

School children were able to meet the PA recommendations if they made appropriate use of a range of available opportunities, such as commuting, recess, playing outdoors, and physical education lessons. In France, the Intervention Centered on Adolescents' Physical activity and Sedentary behavior (ICAPS) has demonstrated the feasibility of implementing a multilevel PA intervention program in adolescents [10]. ICAPS was associated with a significant improvement of activity patterns, indicating that it is a promising approach for modifying the long-term PA level of adolescents, and a way to prevent excessive weight gain in non-overweight adolescents [11,12].

Another option is to use unstructured free play, without any given tasks or goals to promote physical activity among young individuals [13,14].

This study was hence designed to evaluate the efficiency of a sports kit that provides access to sports in a safe and recreational way that we named "Le Sport Ça Me Dit". This kit was developed in 2008, and it is used in more than 1,200 towns throughout France. It comprises a box that contains the equipment needed to set up six sporting activities (for sports that are rarely practiced in France).

The main aim of this study was to evaluate the short- and medium-term effect of the sports kit on children's level of physical activity. It's impact on the duration of sleep and sedentary times (e.g. screen time) were considered as secondary objectives.

\section{Methods}

This study was conducted in the Flandre Lys Community of Towns, a joint local authority group in Northern France (with a total of 38,932 inhabitants) that has been implementing the "Preventing Obesity in Children Together” (“Ensemble Prévenons l'Obésité Des Enfants" or EPODE) methodology for over 20 years. The EPODE methodology consists of concrete initiatives, at both national and local levels, that foster better and more balanced eating habits and greater physical activity in everyday life, in a sustainable way [15].

\subsection{Participants}

Six out of eight towns in the Flandre Lys Community of Towns agreed to participate in the study.

In total, 213 children who were 8 to 11 years of age (comprising 103 boys and 110 girls) were divided into 2 non-randomized groups (the groups were divided according to the constraints of the towns): an Action Group and a Control Group. To ensure homogeneity of the populations studied in terms of the French socio-economic classification (SEC), each town formed an Action group and a Control group. The only exception to this was the town of "Haverskerque", which due to local constraints only formed a Control group. All parents approved of the study design and they signed an informed consent form allowing participation by the children.

\subsection{Study Design}

This study was a 17-week open controlled study, with two parallel groups (Figure 1). All children received a Withings pulse $\mathrm{OX}{ }^{\circledR} \quad 3 \mathrm{D}$ accelerometer wristband (Withings, Issy Les Moulinaux, France) for a three-week trial period. The children were then divided into two groups for 7 weeks (period 1): (i) Action groups with two recreation sessions of 45 minutes per week using the kit (comprising six different activities) (except for the town of Fleurbaix which used the kit for one session of 90 minutes per week) and (ii) Control groups that did not have access to the kit. No use of the kit was provided to either of the groups for the subsequent 7 weeks (period 2). This study was conducted between January and April of 2015.

The sports kit "Le Sport Ça Me Dit” used in this study consists of a box that contains the equipment needed to play the following six different sporting activities: archery, street golf, kin-ball, soccer, street hockey, and tennis. User instructions provide adapted activity rules to remove any notion of performance assessment and to develop sporting values in children, such as sharing, respect, and tolerance. Moreover, the kit is suitable for use in essentially any setting by facilitators who are not specialized in sports. The kit was used during after-school time (designated as extracurricular activities in France). It was moderated by facilitators or a sports teacher.
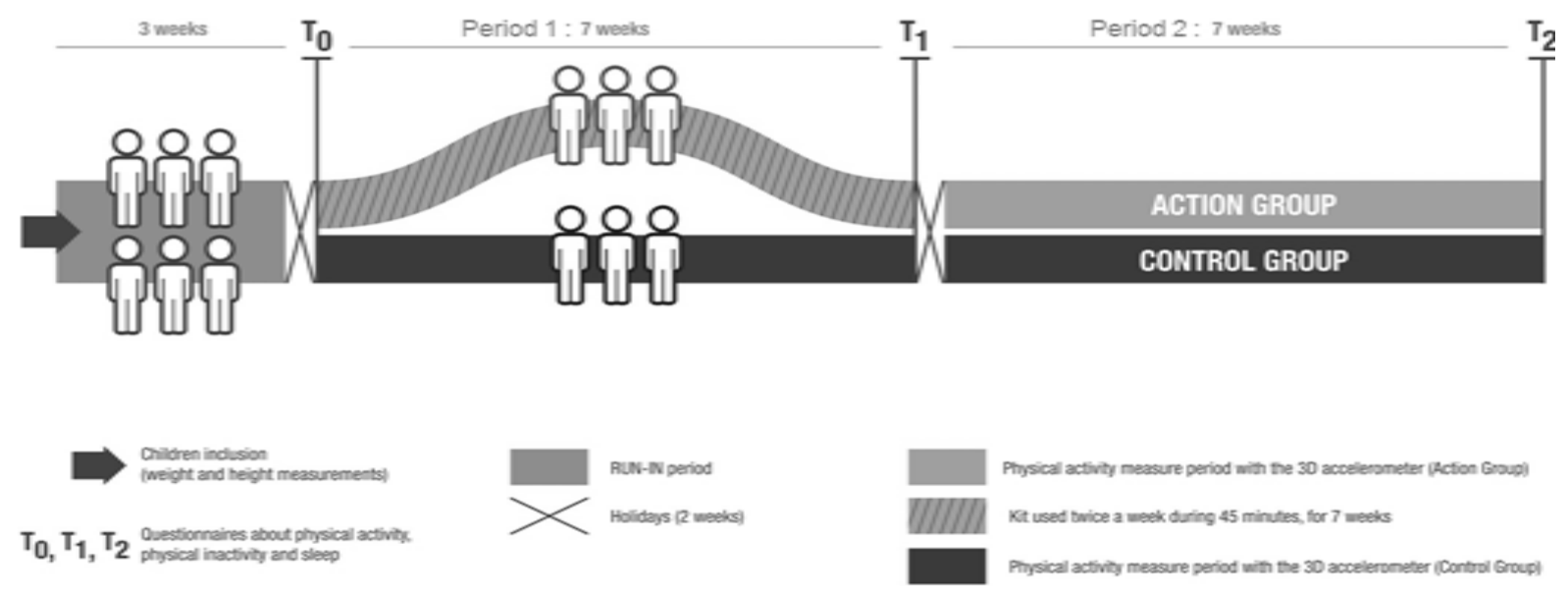

Figure 1. Study design 


\subsection{Personal Data}

The professions of the parents were matched with their corresponding French socio-economic classification (SEC) according to the nomenclature of the French National Institute for Statistics and Economics Studies (INSEE), the French agency for statistics.

Socio-economic classifications were divided into two main groups: SEC+ (businesspeople, trades and craftsmen, executives, intellectual professionals and intermediate professionals); and SEC- which comprises the other classes (e.g. farmers, employees, workers, and those not in the work force).

\subsection{Anthropometric Measurements}

Before the trial period, to set the accelerometer wristbands parameters, healthcare professionals measured the weight and the height of each child. The body weights of the clothed participants (without coats or shoes) were measured using an electronic scale. Their heights were measured using a telescopic height-measuring instrument after removal of the participant's footwear.

Their Body Mass Index (BMI) was calculated as their body weight divided by the square of the individual's height. Being overweight was defined according to the International Obesity Task Force gender-age cutoffs [16]. Overweight and obesity have been grouped together as "overweight" in order to increase the size of this group.

\subsection{Physical Activity Measurements}

\section{Using an Accelerometer}

A Withings pulse $\mathrm{OX}{ }^{\circledR} 3 \mathrm{D}$ accelerometer wristband (Withings, Issy Les Moulinaux, France), was used to record the daily steps number, physical activity, distance travelled, and height difference. The Withings device has been validated for counting of steps and MVPA measurements [17]. Participants were instructed to wear this device (e.g. on their wrist or belt) during all waking hours, except when engaging in water-based activities and when recharging it overnight. The accelerometer wristband data were complied once a week at each school by dedicated staff during the 14 weeks of the study using an IPOD ${ }^{\circledR}$ (MacIntosh Model A1509 Designed by Apple, California, US). In case of a malfunction, the dysfunctional wristband was replaced.

The activity output that was measured included the number of steps and the time engaged in light, moderate, and vigorous physical activity. Data for activity counts and steps were recorded each minute for up to one week. Both the activity count and the step data were extracted by the software owner (Withings ${ }^{\circledR}$ ) using a proprietary signal-filtering algorithm and times. These two related quantities measure physical activity movements associated primarily with locomotion.

Published thresholds were used to classify light physical activity (1000<LPA<3000 counts/minute, 1.5 - 3 METs), moderate and vigorous physical activity (MVPA>3000 counts/minute, >3METs). These thresholds are based on calibration research developed by the software owner (Withings ${ }^{\circledR}$ ).

\section{Based on the questionnaire (QAPE)}

The children were given a validated questionnaire [18] in regard to physical activity (QAPE) so as to assess the variety of their active behaviors and physical activity habits, the intensity of the physical activity, and their screen time habits. Questions were added to the QAPE questionnaire to assess sleep duration and sedentary time spent watching a TV or a computer screen. The QAPE was administered three times during the study to all of the children: at the beginning (T0), during the study (T1), and at the end of the study (T2). The QAPE was completed by the children with an adult supervisor during the time dedicated to extracurricular activities (45 minutes), except for the town of "Fleurbaix", where due to local constraints the questionnaire was administered by parents.

For the Action group, a questionnaire regarding kit use was administered to children by parents during period 2 of the study.

Table 1. Variables studied

\begin{tabular}{|c|c|c|}
\hline Qualitative Variables & Quantitative variable & Units / comments \\
\hline Age & & Years \\
\hline \multirow[t]{2}{*}{ Gender } & & Female / Male \\
\hline & BMI & $\mathrm{Kg} / \mathrm{m}^{2}$ \\
\hline BMI Categories & & $\begin{array}{l}\text { Normal weight } \\
\text { Overweight (Overweight and Obese) }\end{array}$ \\
\hline CSP & & 9 divided into 2 classes : CSP+ / CSP- \\
\hline Towns & & 6 towns \\
\hline \multirow[t]{5}{*}{ School } & & 7 schools \\
\hline & Variety of PA score & Out of 48 \\
\hline & Intensity score & Out of 7 \\
\hline & Sedentary score & Out of 28 \\
\hline & Number of hours of sleep & \\
\hline \multicolumn{3}{|l|}{ Questionnaire (19 items) } \\
\hline \multirow[t]{3}{*}{ 3D accelerometer wristband Withings } & Number of steps & $\begin{array}{l}\text { Average steps per day } \\
\text { Average steps per weekday } \\
\text { Average steps per day on weekends }\end{array}$ \\
\hline & Time spent engaged in light PA & In minutes per day \\
\hline & Time spent engaged in moderate or vigorous PA & In minutes per day \\
\hline
\end{tabular}


The QAPE questionnaire was designed to provide 3 "scores": (i) the "variety of physical activity score" on a scale of 0 to 48, assessing active behavior (e.g. walking to school, practiced physical activity, movements, etc.), (ii) the "sedentary score" on a scale of 0 to 28, assessing the sedentary time spent watching a TV or a computer screen, and (iii) the "intensity score" on a scale of 0 to 7 , assessing the intensity of the performed physical activity. Details of how these are calculated have been published by Tessier and collaborators in 2007 [18]. Sleep time was calculated in hours; and the time spent watching a TV, computer, or tablet was in calculated in minutes. Qualitative and quantitative data were identified using questionnaires to measure the children's interest in the kit and the ease of using the sports kit by the facilitators. Qualitative variables and quantitative variables were studied (Table 1).

\subsection{Statistical Analyses}

The accelerometer data were converted into daily data using Excel software. The moderate and vigorous physical activity (MVPA) data were added. Data below 1,000 steps per day were considered outliers according to the methodology recommended for missing data when using a pedometer [19]. LPA and MVPA data corresponding to a threshold lower than 1,000 steps per day were also considered to be outliers.

Since the initial population was not homogenous per group in terms of gender; an adjustment was made by combining a random process (i.e. systematic sampling) and an empirical approach.

The adjusted sample was composed of 180 children (92 girls and 88 boys, mean age: 9.95 years). All statistical analyses were performed using Microsoft Excel 2010 and a data analysis software (XLSTAT). Analyses of the characteristics of the study population were performed on the initial sample (213 children). Basic sorting, cross sorting, and box plots were used.

Inferential statistical tests were performed on the adjusted sample to compare the 2 groups (Action and Control) for each variable and their progression. $\chi^{2}$ tests, analyses of variance (ANOVA), and Mann-Kendall tests were used. A P-value of less than 0.05 was considered significant. Missing data were not replaced. For ANOVA, missing data were treated by removal of observations.

Steps number and scores of the Action group using the sports kit 1 session of 90 min per week were not significantly different from the Action group using the kit 2 sessions of 45 min per week (steps number: $\mathrm{p}=0.190$; variety of physical activity score: $\mathrm{p}=0.069$; sedentary score: $\mathrm{p}=0.469$;intensity score $\mathrm{p}=0.125$ ). We therefore subsequently grouped them together.

\section{Results}

\subsection{Baseline Characteristics}

The study participants comprised 110 girls and 103 boys, with a mean age of 9.9 years (range 7-12 years) and an overweight prevalence of $17.4 \%$. The frequency of being overweight was similar for the boys and the girls.
Physical activity: 64\% of the children in the study travelled to school by car or by bus. Boys were significantly more active than girls during recess periods $(p=0.001)$. Forty percent of the children in the study engaged in at least one or more physical activity outside of school on a daily basis. The mean number of steps per day for the boys was 8,405 (SD = 1,732), which was significantly higher than for the girls who recorded a mean of 7,465 (SD $=1616)$ steps per day $(p<0.0001)$. The participants were significantly more active on weekdays $(8,493$ steps, $\mathrm{SD}=1,834)$ than on weekends $(6,424$ steps, $\mathrm{SD}=2,265)(\mathrm{p}<0.0001)$. Moreover, a significant change in activity was observed between periods 1 and 2 . In terms of watching television and sleeping: a third of the children in the study reported watching television every morning on schooldays. Half of the children in the study reported watching television every night on schooldays. Twenty-eight percent of the children in the study had a television in their bedroom. On average, children in the study reported watching TV for $1 \mathrm{~h} 30$ per day on weekdays and $2 \mathrm{~h} 10$ on weekends.

Ninety-six percent of the children in the study reported sleeping more than 9 hours per night on weekdays.

Study characteristics of the adjusted population of 92 girls and 88 boys, with a mean age of 9.95 years (range 7.39 - 11.39 years) are presented in Table 2. It can be seen that the baseline characteristics are similar between the two groups (Table 2).

Table 2. Baseline characteristics by group

\begin{tabular}{|c|c|c|c|c|}
\hline \multirow[t]{2}{*}{ Characteristics } & \multicolumn{2}{|c|}{ Control $(n=107)$} & \multicolumn{2}{|c|}{ Action (n=73) } \\
\hline & Mean & SD & Mean & SD \\
\hline Age, years & 9.94 & 0.83 & 9.97 & 0.86 \\
\hline \multirow[t]{2}{*}{ BMI, kg/m² } & 17.75 & 2.66 & 17.20 & 2.63 \\
\hline & \multicolumn{2}{|c|}{$\%$} & \multicolumn{2}{|c|}{$\%$} \\
\hline Males & \multicolumn{2}{|c|}{51.4} & \multicolumn{2}{|c|}{45.2} \\
\hline Overweight & \multicolumn{2}{|c|}{22.4} & \multicolumn{2}{|c|}{10.95} \\
\hline CSP + (Father) & \multicolumn{2}{|c|}{62.75} & \multicolumn{2}{|c|}{60.56} \\
\hline CSP - (Father) & \multicolumn{2}{|c|}{37.25} & \multicolumn{2}{|c|}{39.44} \\
\hline
\end{tabular}

\subsection{Data from the Questionnaire (QAPE): Physical Activity, Screen Time, and the Time Spent Sleeping}

The children in the Action group increased their overall active behavior between the T0 and the T2 interview. Thus, "the variety of physical activity score" increased significantly ( $p=0.021)$ vs. the Control group (Figure 2 ).

Based on the average for the 3 interviews, the intensity of activity (i.e. "the intensity score") was significantly higher for the Action group vs. the Control group (Figure 3) $(p=0.038)$. Use of the kit had no effect on the amount of screen time reported by the children (i.e. "the sedentary score”). For the three scores, no significant gender difference was observed between the groups. There was no significant difference for the reported duration of sleep between the Action group and the Control group.

Based on the second interview $\mathrm{T} 1$, the time spent watching TV was greater for the Action than for the Control group $(\mathrm{p}=0.039)$. 


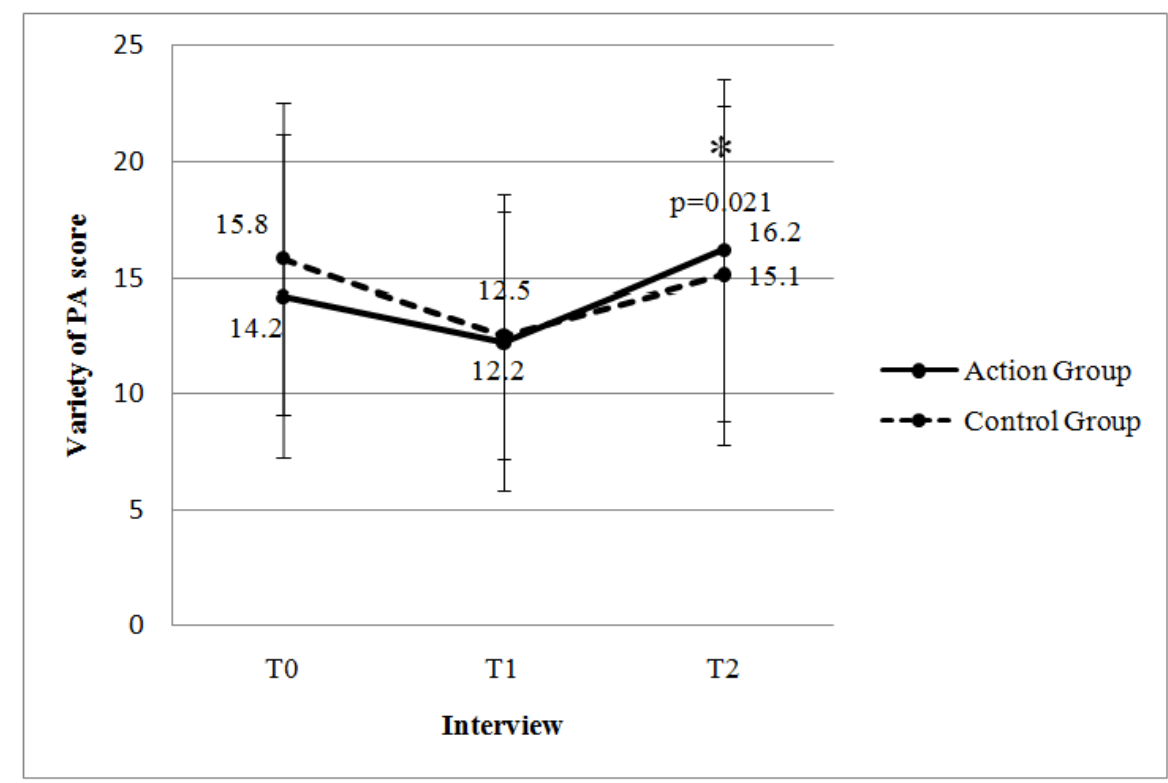

A P-value is provided for the effect of the PA initiative (changes in variety between $\mathrm{T} 0$ and $\mathrm{T} 2$ for the Action group vs. the Control group)

Figure 2. Variety of physical activity score by group $\left({ }^{*} \mathrm{p}<0.05\right)$

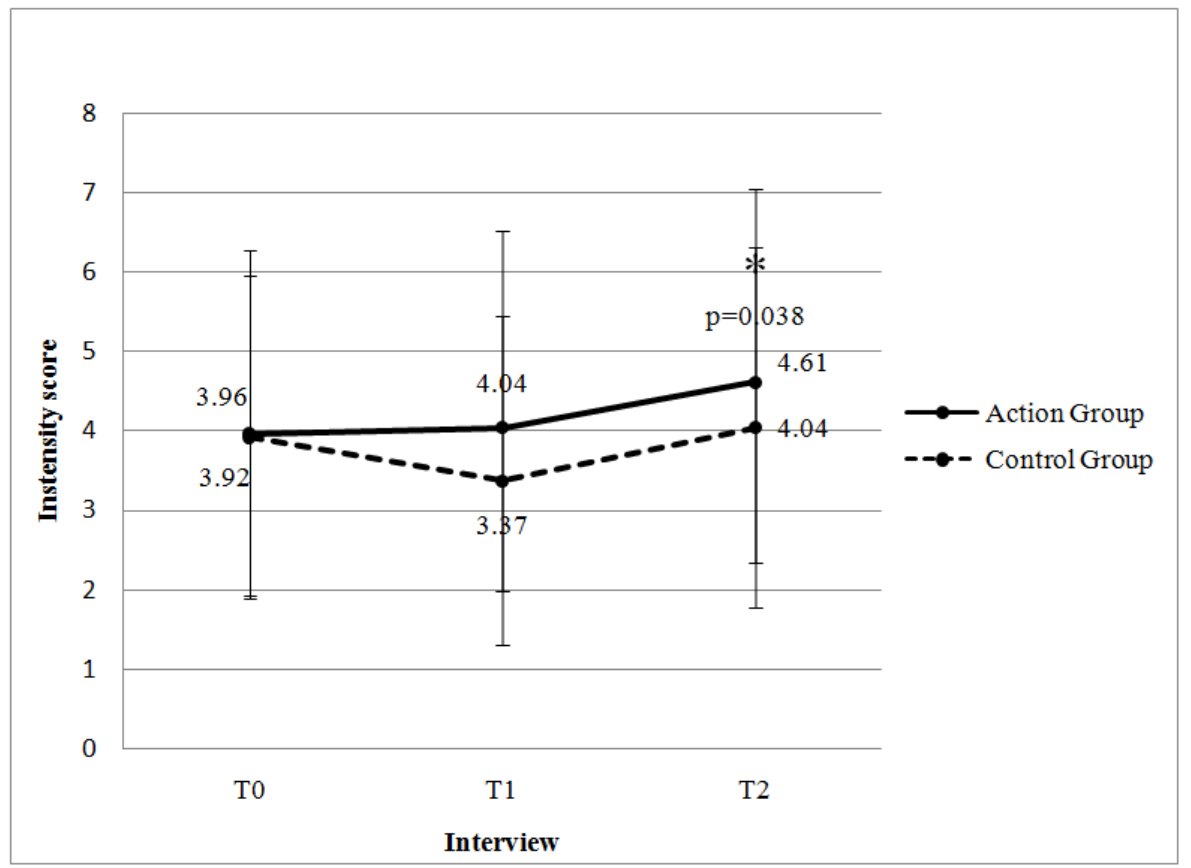

A P-value is provided for the effect of the PA initiative (changes in intensity between T0 and T2 for the Action group vs. the Control group.

Figure 3. Intensity score by group $\left({ }^{*} \mathrm{p}<0.05\right)$

Thus, although no significant difference was observed in terms of screen time and the duration of sleep, children using the sports kit exhibited a higher overall level of active behavior and a higher intensity of activity as compared to the Control group.

\subsection{Data from the Accelerometer: Steps Number and Physical Activity}

\section{Steps number}

The Action group performed more steps in period $1 v \mathrm{~s}$. the Control group $(p=0.025)$. However, this significant difference was not seen in period $2(p=0.672)$ (Figure 4). In terms of gender, boys of the Action group took more steps than did boys in the Control group ( $p=0.038)$ in period 1, but this significant difference was not seen in period 2. Also, this effect was not seen in either period with girls.

Children from SEC+ mothers or fathers exhibited a higher steps number ( $p=0.004$ (mother) and $p=0.005$ (father)) compared to children with SEC- backgrounds.

The effect of the kit was, however, not associated with the parent's professions.

Analysis of the subpopulation of overweight children who used the sports kit revealed a significant increase in the steps number for both periods compared with the Control overweight group $(\mathrm{p}=0.018$ and $\mathrm{p}=0.012$, respectively) (Figure 5). 


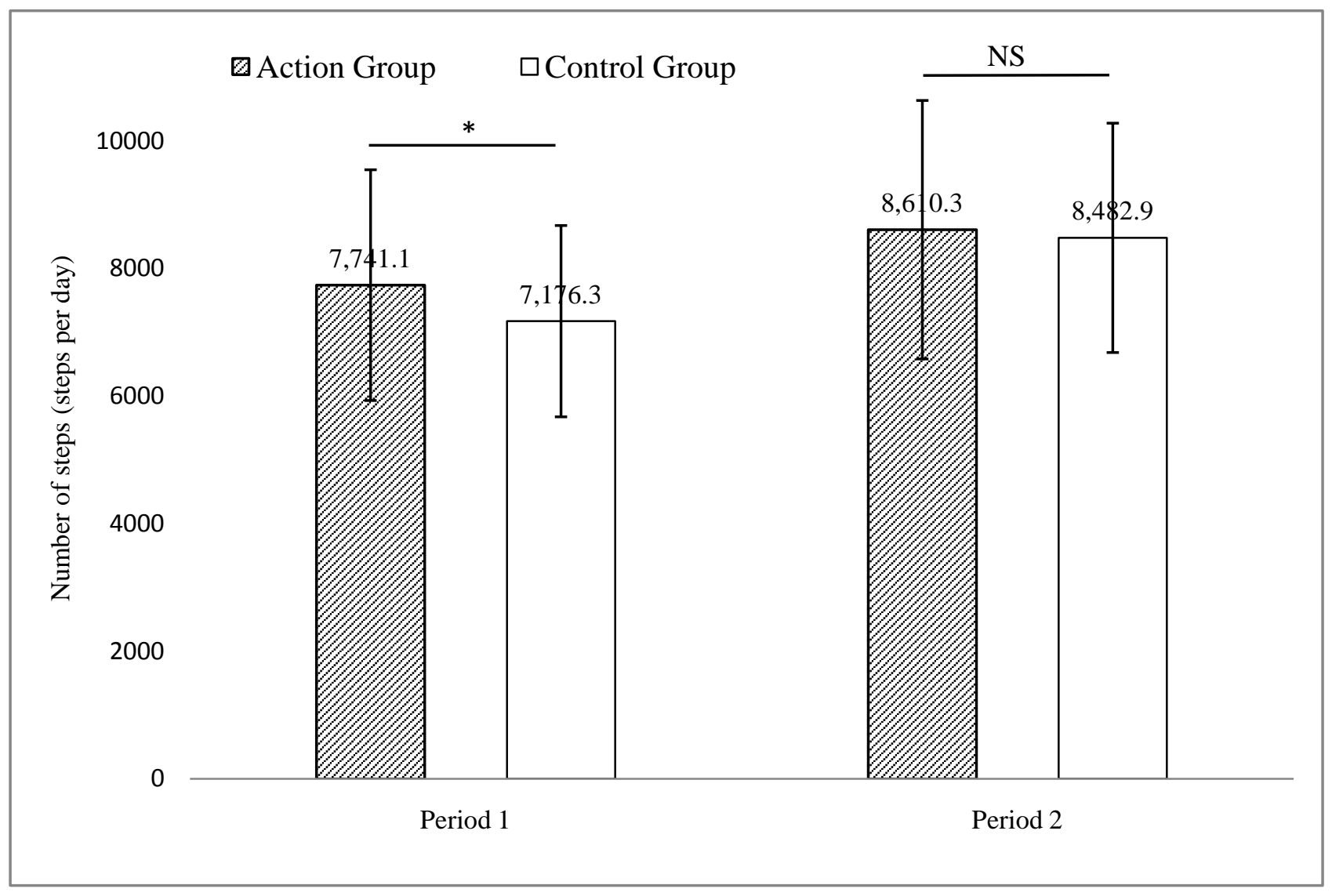

Figure 4. Number of steps by group $\left({ }^{*} \mathrm{p}<0.05\right)$

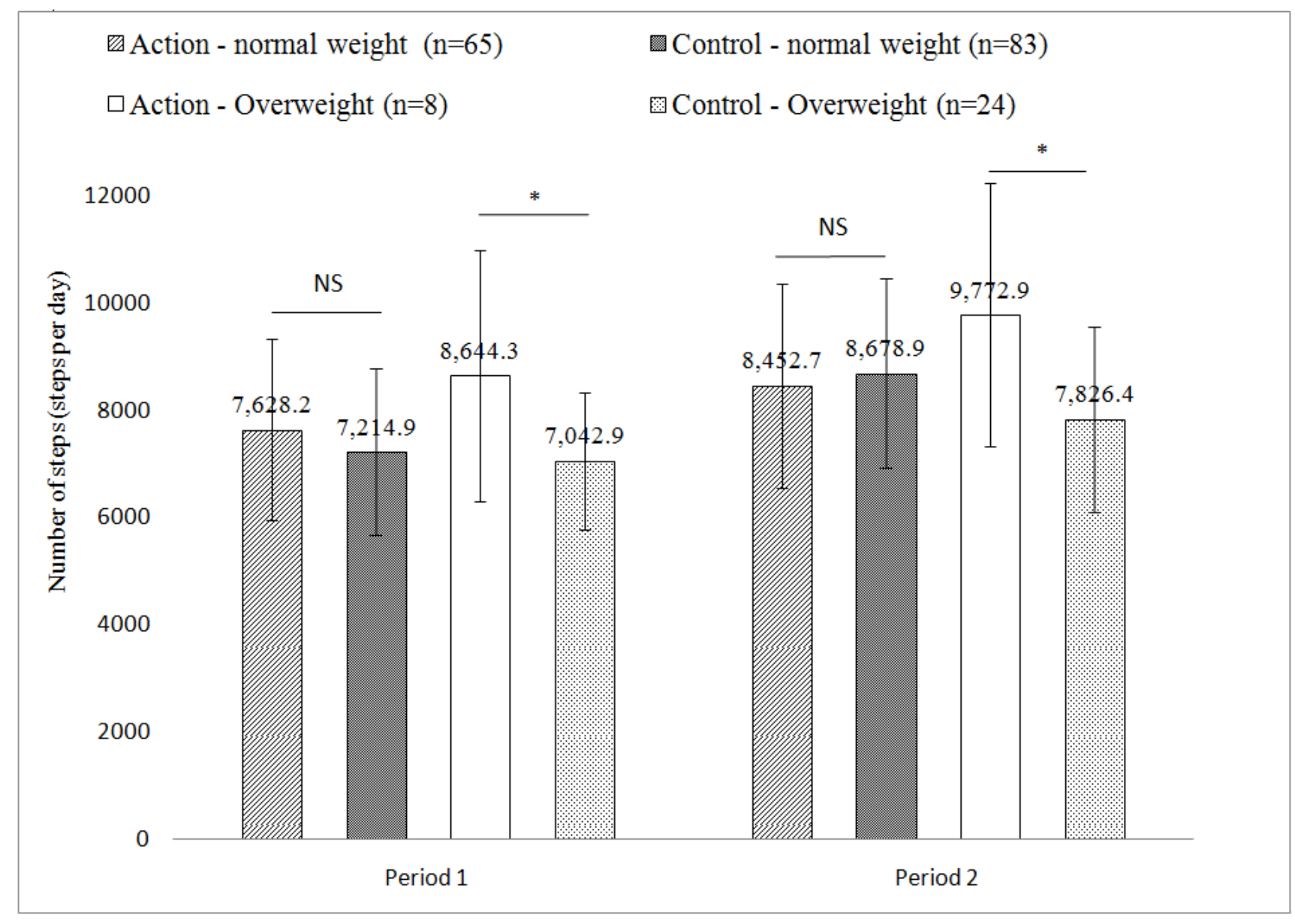

Figure 5. Number of steps according to weight groups $\left({ }^{*} \mathrm{p}<0.05\right)$ 


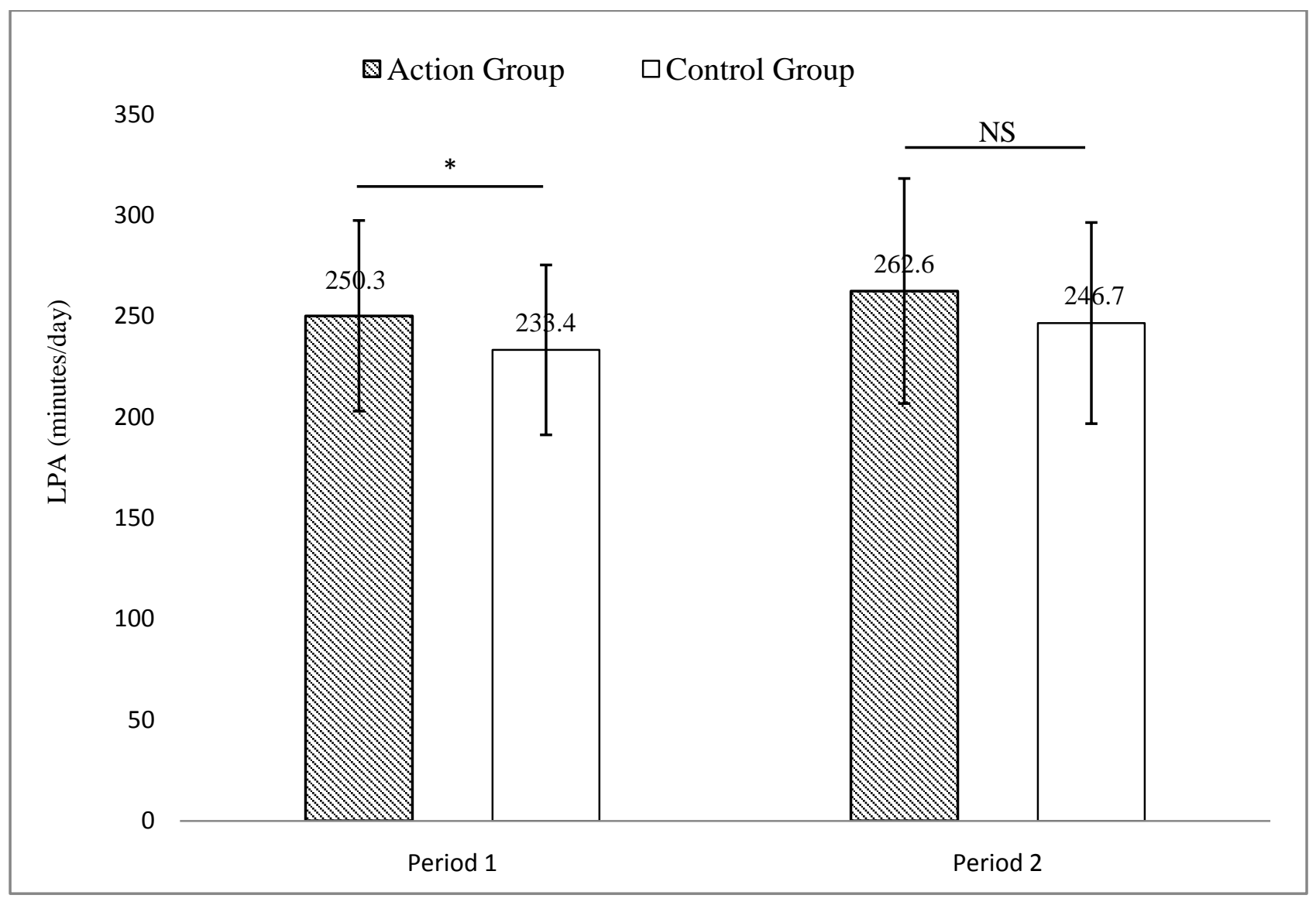

Figure 6. Light physical activity (LPA) by group $\left({ }^{*} \mathrm{p}<0.05\right)$

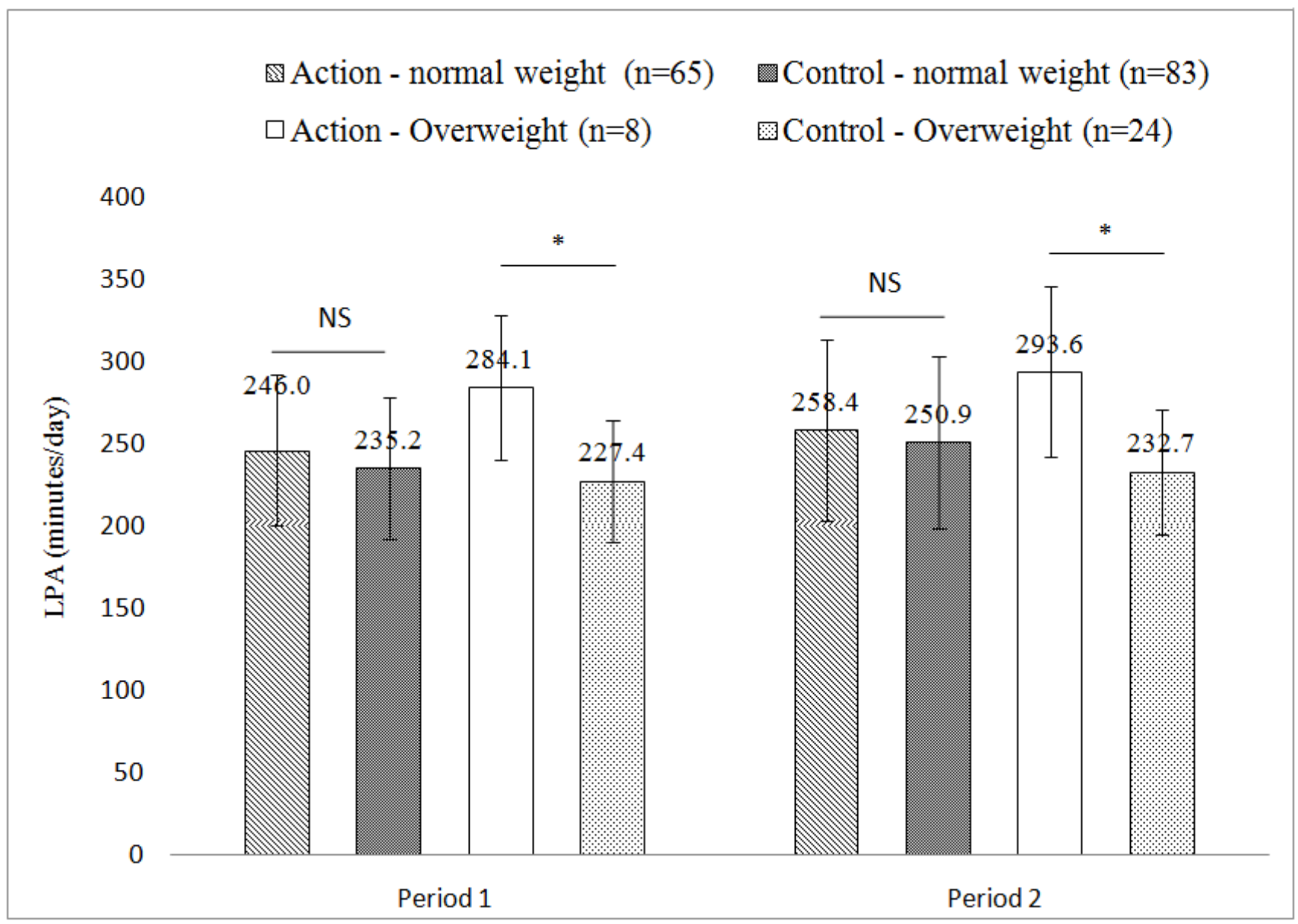

Figure 7. Light physical activity (LPA) according to weight groups $\left({ }^{*} \mathrm{p}<0.05\right)$ 


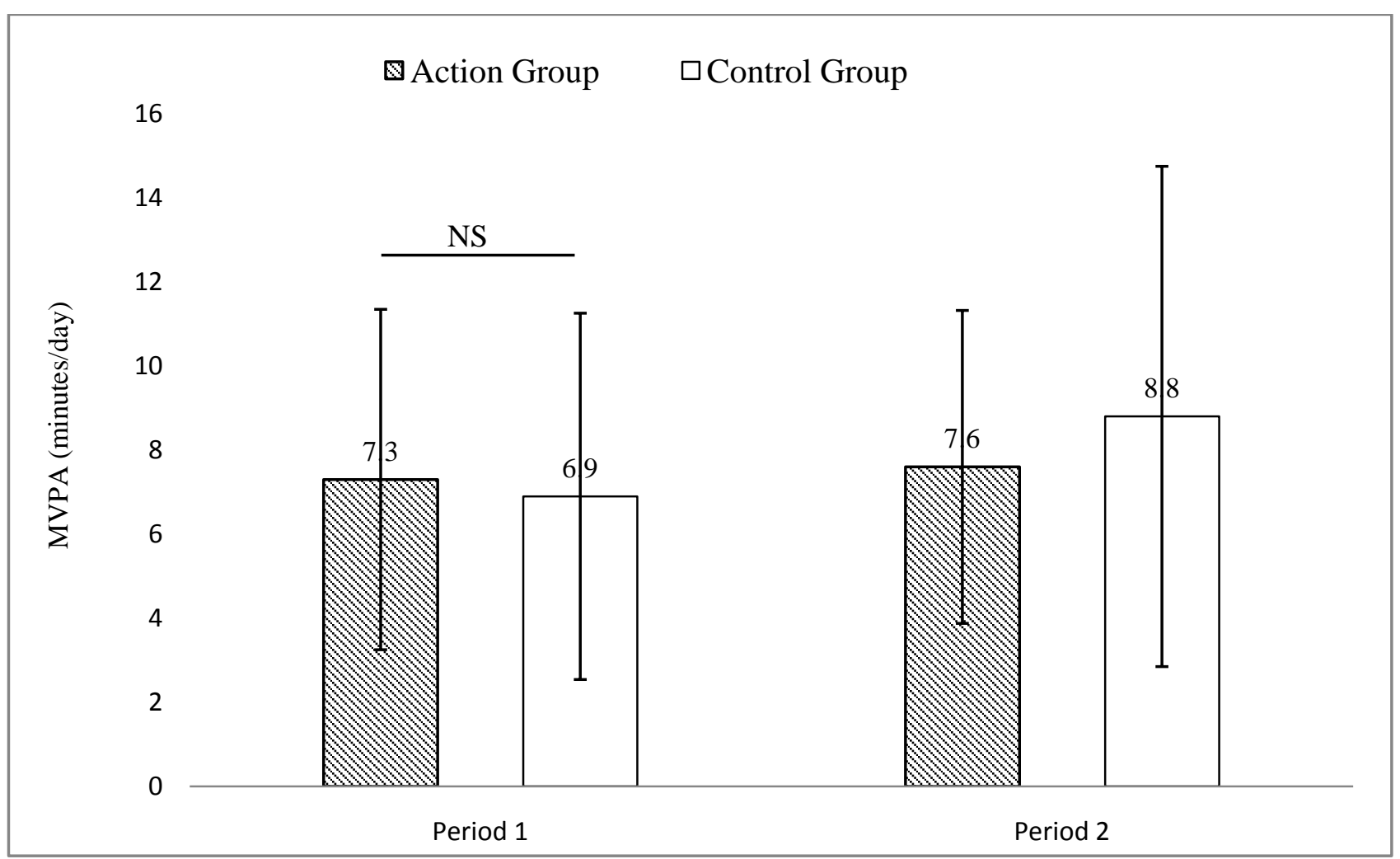

Figure 8. Moderate and Vigorous Activity (MVPA) by group

\section{Physical activity}

The data indicates that children in the Action group performed more total physical activity in period 1 compared to children in the Control group $(\mathrm{p}=0.011)$. However, this effect on physical activity was not seen in period $2(\mathrm{p}=0.074)$.

In regard to light physical activity, similar results were observed. Thus, children in the Action group performed more LPA in period 1 compared to children in the Control group ( $\mathrm{p}=0.014)$. As for total physical activity, the effect did not persist in Period $2(p=0.057)$ (Figure 6). However, we observed an interesting effect for the population of overweight children. The overweight children of the Action group performed more LPA in both periods compared to the overweight children of the Control group $(\mathrm{p}=0.010$ and $\mathrm{p}=0.025$, respectively) (Figure 7). Confirmation of this result would provide interesting insights in regard to overweight children. We also found that the effect of the kit on LPA was not associated with the parent's professions and gender.

In terms of moderate and vigorous physical activity, no significant difference was observed between children in the Action and the Control groups, whether in period 1 ( $\mathrm{p}$ $=0.532)$ or in period $2(\mathrm{p}=0.137)$ (Figure 8$)$. However, the Control group exhibited a significant increase in time engaged in MVPA between period 1 and $2(\mathrm{p}=0.022)$. The effect of the kit on the level of MVPA was not significantly associated with gender, the parents' profession, or with being overweight.

\section{Discussion}

Our results demonstrate that the effects of the sports kit on PA were: (i) use of the kit resulted in an increase in the daily steps number and LPA without any compensatory increase in the amount of time spent engaged in sedentary activities, as measured by questionnaire. These data take into account the two days per week using kit as well as the other days of the week. (ii) use of the kit resulted in an increase in the daily steps number and LPA in overweight children even after terminating its use, without any compensatory increase in the amount of time spent engaged in sedentary activities.

\subsection{Baseline Characteristics}

It can be seen that being overweight is less common among children in the study (17.4\%) than among children in general in the region (20.7\%), and this may be due to the EPODE program.

The study population exhibited a mean value of 8,405 steps/day. Although steps number is not a marker of all PA, it is nonetheless a good indicator, and it fell below the recommended level of 12,000 steps/day for girls and 15,000 steps/day for boys [20], and it corroborates the following data on MVPA. We also found that the time spent engaged in MPVA was low for our study population (with a mean of 7.6 minutes per day). While several other studies using an accelerometer to measure PA found much higher MVPA levels [21], this was not always found to be the case [9]. Based on the international recommendation of at least 60 minutes of MPVA per day, only $10 \%$ of girls in the UK and $1 \%$ of girls in France (aged $9.9 \pm 0.9$ years) achieved this intensity for the recommended 60 min per day [22], or met the current PA guidelines for youths [9]. Although it is difficult to compare characteristics of our population with those of other studies of PA in children that used different methodologies to quantify PA and to treat outlier data. 
The increase in our population's daily steps number between period 1 and period 2 could be due to a seasonal effect. Some research has shown that weather patterns and daylight hours can have an effect on physical activity [23]. Relative to winter months, physical activity has been shown to peak in summer months [24] due to longer daylight hours and more favorable weather conditions $[25,26]$.

It is unclear why "variety of PA score" (as assessed by a questionnaire) decreased at $\mathrm{T} 1$ for both groups, but it may be also due to bad weather at this time of the measure.In our study, the population's daily steps number (as measured by an accelerometer) was lower on weekends. Other studies have confirmed this finding, and have implicated, among other factors, the closure on weekends of the school infrastructure dedicated to physical activity $[27,28,29,30]$. By contrast, studies regarding the role of access to infrastructure dedicated to physical activity outside of school hours and on nonschool days showed that such access led to a significant increase in the level of PA in children [12].

The gender differences observed in our study on physical activity (i.e. steps number measured by an accelerometer) and on activity during recess (as assessed by the questionnaire) are in agreement with prior studies. These studies reported lower levels of female physical activity, with this difference starting in infancy and continuing over the course of their lives. Accordingly, boys exhibited higher total levels of physical activity than did girls, and also specifically during school recess periods [31,32].

As sedentary lifestyle is considered to be a risk factor for cardiometabolic diseases, it is very important to fully define this type of behavior. Sedentary behavior can readily be measured by determining cumulative screen time (i.e. time spent watching TV, video games, tablet or computer screens). It has been reported that screen time is associated with enhanced adiposity [33], and insulin resistance [34]. Furthermore, it has been demonstrated that physical inactivity that is linked with high sedentary behaviors can lead to metabolic disorders such as insulin resistance [7]. In our study population, the total time spent in front of a screen (as measured by the questionnaire) was high (151 min on weekdays and 223 min on weekends), thus indicating that most children exceed the guidelines that recommend no more than 2 hours of recreational screen time per day $[35,36]$. One explanation is that half of the children in the study watch TV every evening.

\subsection{Effects of the Kit}

Previously made observations suggest that the time engaged in MVPA, as well as overall physical activity is associated with a healthier cardiometabolic profile in young individuals [37]. Although the time engaged in MPVA did not change in the Action group, the number of steps taken in a day and the level of LPA increased significantly for this group, and the total level of PA increased significantly during the action period (P1). It is unclear why MVPA increased only in the Control group at $\mathrm{P} 1$, although it may reflect stimulation by the accelerometer wristband.

For the Action group, the number of steps taken per day and the level of low-intensity PA increased, while there was no change in the sedentary time during the experimental period. Compensatory responses with a decrease in energy expenditure on spontaneous physical activity after prescribed physical activity have been reported, mainly in obese subjects [38,39]. Rowland [40] first described this phenomenon, using the term 'ActivityStat', and proposed that the control of physical activity was operated centrally according to a set point of energy expenditure. This theory predicts that more activity at any given time will be compensated for by less activity at another time so as to preserve the individual's set point. Fremeaux et al. [41] demonstrated this phenomenon in school-aged children, but it has also been reported to occur with adults [42].

Our result, showing an increase in total PA (LPA+MVPA), measured by an accelerometer, that can largely be explained by increased spontaneous lowintensity PA in the Action group, without a compensatory increased time spent engaged in sedentary activities, measured by the questionnaire (with the exception of time spent in front of a screen in $\mathrm{T} 1$ for the Action group, but with no change in the overall sedentary score), is an important finding. This data takes into account the days on which the activity occurred, as well as the other days of the week.

On the other hand, children are also exposed to other kinds of sedentary behaviors during the day, defined as non-screen sedentary behaviors (e.g. time in the classroom, commuting to school) that were not accounted for in the present study. It is however unlikely that there were changes in these times that are independent of the child. Collectively these data show that a period of recreational PA does not subsequently result in decreased PA or increased sedentary time.

The increase in the number of steps and in LPA was no longer seen in the medium term (i.e. in Period 2, when the kit was no longer available), suggesting that availability of the kit needs to be longer in order for there to be a noticeable longer term change in behavior. Two studies that were able to show a successful outcome in terms of increased physical activity involved either a six month or a four year intervention $[12,43]$.

There is evidence, however, that supports public health interventions aimed at increasing PA during after-school time (i.e. as extracurricular activities). Others studies have identified the school itself as a key setting for the promotion of physical activity in young individuals $[12,43,44]$. The results with overweight children are in line with these studies.

The effect of the sports kit on the overweight subgroup in the short- and medium-term is noteworthy: it increased walking and spontaneous light PA in P1, and this increase in PA was maintained in P2. A post-kit effect was not observed in children with normal weights. In the current study, overweight children complied well with the prescribed use of the sports kit. This observation suggests that the sports kit (when used twice/week or $90 \mathrm{~min} /$ week) is a feasible and effective way for overweight girls and boys to increase their level of exercise. One explanation for this may lie with the fact that since the kit is a non-competitive device it could increase their willingness to be more active. As is the case with obese adolescents, this can lead to a substantial impact on health, as Lee et al. 
[45] have demonstrated the usefulness of exercise on its own (without caloric restriction) as a strategy for reducing obesity-related health risks.

Our study has several limitations; firstly a lack of child compliance was noted: only 12 children out of the 213 (5.6\%) wore their wristband accelerometer every single day $(11.7 \%$ of the children wore their wristband accelerometer every single day for the first period, and $13.6 \%$ of the children wore their wristband accelerometer every single day for the second period). Over the two periods, $32 \%$ of the data was missing, and for $53 \%$ of the children this amounted to more than $75 \%$ of the measurement data, with no significant difference between the Action and the Control group $(\mathrm{p}=0.285)$. However, as the long period of data collection (45 days) allowed measurements to be repeated throughout the experimental period, and the results of the analysis revealed no significant differences between the two groups in terms of compliance, the collected data was nonetheless adequate for the intended analyses. Secondly, we noted that the children tended to require assistance with answering the questionnaire, and some of the questions were difficult for their age, such as the quantification of screen time. A key issue for studies on PA is the need to obtain objective measurements. Thirdly, it should be noted that another limitation of this study was the low number of children in the overweight subgroup ( $n=8$ in the Action group and $n=24$ in the Control group), and the results should hence be confirmed through a larger study, including measurements of adiposity.

This study also had substantial strengths, however. Firstly, two methods were used to quantify physical activity: a questionnaire and an accelerometer. Although the children needed assistance to answer some of the questions, use of the questionnaire allowed additional information to be collected (e.g. screen time with TVs, computers, tablets, and the variety of active behaviors, etc.), and compliance was excellent (a 100\% response rate at $\mathrm{T} 0$, a $97 \%$ response rate at $\mathrm{T} 1$, and a $94 \%$ response rate at T2). Secondly, use of accelerometers provides an advantage over self-reported questionnaires. They are used in large epidemiological studies of children's activity [46].

Thirdly, feedback on the kit from the children was very positive: the children were keen to use it, and they exhibited a willingness to be more physically active.

The children's Action group answered as follows to these questions:

- "Does the kit make you want to move more?" - 86\% yes,

- "Did you want to keep the kit during the extracurricular activities?” - 88\% yes,

- "Did you discover a new activity with the kit?" - 50\% yes.

\section{Conclusion}

In conclusion, our study shows that the sports kit is effective at increasing children's LPA, physical activity, the number of steps that they take each day, and their motivation to be more active without a compensatory increase in sedentary time. This positive effect was seen in the short-term, although it was not maintained after seven weeks in the children with normal body weights. There appears to be a mid-term positive impact of the use of the kit among overweight children that warrants further investigation. If it is confirmed with a higher number of children, it provides interesting insights in regard to these children. When used twice a week, a device offering a variety of sporting activities, with non-competitive objectives, can significantly increase PA activity levels over a seven week period. Availability of this device could hence have a significant impact on overall health. The lower level of PA observed with children on weekends suggests that public strategies to promote PA may be needed on weekends. An example of this is the kit "Le Sport Ça Me Dit”, as it is a useful and easy-to-use device that can be implemented as a public strategy to promote PA in children on weekends.

\section{Acknowledgments}

We thank elected officials from the Community of the Towns of Flandre Lys for having supported the establishment of the study on the territory.

\section{Source of Funding}

Research related to this manuscript was funded by a grant from the Coca-Cola Foundation and technical support was received from Withings.

\section{Conflicts of Interest}

A. $\mathrm{C}^{5}$ and A.N are employed by Withings. JMB and JM are employed by EPODE. The others authors declare no conflict of interest.

The content of the present manuscript reflects only the authors' views.

All authors read and approved the final manuscript.

\section{Ethical Standards Disclosure}

The CNIL declaration of this study was in accordance with French regulations. All parents approved of the study design and they signed an informed consent form allowing participation by the children.

\section{References}

[1] Hallal PC, Victora CG, Azevedo MR, et al. (2006). Adolescent physical activity and health: a systematic review. Sport. Med 36, 1019-1030.

[2] Janssen I \& Leblanc AG. (2010). AAD.- Systematic review of the health benefits of physical activity and fitness in school-aged children and youth. Int J Behav Nutr Phys Act., 40

[3] Gutin B, Yin Z, Humphries MC, et al. (2005). Relations of moderate and vigorous physical activity to fitness and fatness in adolescents. Am J Clin Nutr 81, 746-750.

[4] Andersen LB, Harro M, Sardinha LB, et al. (2006). Physical activity and clustered cardiovascular risk in children: a crosssectional study (The European Youth Heart Study). Lancet (London, England), 368, 299-304. 
[5] Rizzo NS, Ruiz JR, Oja L, et al. (2008). Associations between physical activity, body fat, and insulin resistance (homeostasis model assessment) in adolescents: the European Youth Heart Study. Am J Clin Nutr, 87, 586-592.

[6] Carson V \& Janssen I. (2011). Volume, patterns, and types of sedentary behavior and cardio-metabolic health in children and adolescents: a cross-sectional study. BMC Public Health 11, 274.

[7] Belcher BR, Berrigan D, Papachristopoulou A, et al. (2015). Effects of Interrupting Children's Sedentary Behaviors With Activity on Metabolic Function: A Randomized Trial. J Clin Endocrinol Metab, 100, 3735-3743.

[8] Plasqui G, Bonomi AG \& Westerterp KR. (2013). Daily physical activity assessment with accelerometers: new insights and validation studies. Obes Rev, 14, 451-462.

[9] Apete GK, Zitouni D, Hubert H, et al. (2012). Compliance of children in northern France with physical activity recommendations. Perspect Public Heal. 132, 81-88.

[10] Simon C, Wagner A, Platat C, et al. (2006). ICAPS: a multilevel program to improve physical activity in adolescents. Diabetes Metab, 32, 41-49.

[11] Simon C, Wagner A, DiVita C, et al. (2004). Intervention centred on adolescents' physical activity and sedentary behaviour (ICAPS): concept and 6-month results. Int J Obes Relat Metab Disord, 28 Suppl 3, S96-S103.

[12] Simon C, Schweitzer B, Oujaa M, et al. (2008). Successful overweight prevention in adolescents by increasing physical activity: a 4-year randomized controlled intervention. Int J Obes, 32, 1489-1498.

[13] Barlow SE \& Dietz WH. (1998). Obesity evaluation and treatment: Expert Committee recommendations. The Maternal and Child Health Bureau, Health Resources and Services Administration and the Department of Health and Human Services. Pediatrics, 102, E29.

[14] Remmers T, Van Kann D, Gubbels J, et al. (2014). Moderators of the longitudinal relationship between the perceived physical environment and outside play in children: the KOALA birth cohort study. Int J Behav Nutr Phys Act, 11, 150.

[15] Borys JM, Le Bodo Y, Jebb SA, et al. (2012). EPODE approach for childhood obesity prevention: methods, progress and international development. Obes Rev, 13, 299-315.

[16] Cole TJ, Bellizzi MC, Flegal KM, et al. (2000). Establishing a standard definition for child overweight and obesity worldwide: international survey. BMJ, 320, 1240-1243.

[17] Ferguson T, Rowlands A V, Olds T, et al. (2015). The validity of consumer-level, activity monitors in healthy adults worn in free-living conditions: a cross-sectional study. Int J Behav Nutr Phys Act, 12, 42.

[18] Tessier S, Vuillemin A \& Briançon S. (2007). Propriétés psychométriques d'un questionnaire de mesure de l'activité physique chez l'enfant scolarisé âgé de six à dix ans: QAPEsemaine. Sci. Sports, 22, 224-231.

[19] Rowe D, Maher M, Raedeke T, et al. (2004). Measuring physical activity in children with pedometers: reliability, reactivity, and replacing missing data. Pediatr. Exerc. Sci., 16, 343-354.

[20] Tudor-Locke C \& Bassett Jr. DR. (2004). How many steps/day are enough? Preliminary pedometer indices for public health. Sport. Med, 34, 1-8.

[21] Guinhouya BC. (2010). [Physical activity of schoolchildren in France. The paradox of a public health priority!]. Rev Epidemiol Sante Publique, 58, 255-267.

[22] Metcalf BS, Voss LD, Hosking J, et al. (2008). Physical activity at the government-recommended level and obesity-related health outcomes: a longitudinal study (Early Bird 37). Arch Dis Child, 93, 772-777.

[23] Reilly T \& Peiser B. (2006). Seasonal variations in health-related human physical activity. Sport. Med, 36, 473-485.

[24] Atkinson G \& Drust B. (2005). Seasonal rhythms and exercise. Clin Sport. Med, 24, e25-34, xii-xiii.
[25] Rich C, Griffiths LJ \& Dezateux C. (2012). Seasonal variation in accelerometer-determined sedentary behaviour and physical activity in children: a review. Int J Behav Nutr Phys Act, 9, 49.

[26] Tucker P \& Gilliland J. (2007). The effect of season and weather on physical activity: a systematic review. Public Health, 121, 909-922.

[27] Brusseau TA. (2015). The Intricacies of Children's Physical Activity. J Hum Kinet, 47, 269-275.

[28] Comte M, Hobin E, Majumdar SR, et al. (2013). Patterns of weekday and weekend physical activity in youth in 2 Canadian provinces. Appl Physiol Nutr Metab, 38, 115-119.

[29] Duncan JS, Schofield G \& Duncan EK. (2006). Pedometerdetermined physical activity and body composition in New Zealand children. Med Sci Sport. Exerc, 38, 1402-1409.

[30] Riddoch CJ, Mattocks C, Deere K, et al. (2007). Objective measurement of levels and patterns of physical activity. Arch Dis Child, 92, 963-969.

[31] Belcher BR, Berrigan D, Dodd KW, et al. (2010). Physical activity in US youth: effect of race/ethnicity, age, gender, and weight status. Med Sci Sport. Exerc, 42, 2211-2221.

[32] Stratton G, Ridgers ND, Fairclough SJ, et al. (2007). Physical activity levels of normal-weight and overweight girls and boys during primary school recess. Obes. (Silver Spring), 15, 15131519.

[33] Carvalhal MM, Padez MC, Moreira PA, et al. (2007). Overweight and obesity related to activities in Portuguese children, 7-9 years. Eur J Public Heal. 17, 42-46.

[34] Atkin AJ, Sharp SJ, Corder K, et al. (2014). Prevalence and correlates of screen time in youth: an international perspective. Am J Prev Med, 47, 803-807.

[35] Tremblay MS, Leblanc AG, Janssen I, et al. (2011). Canadian sedentary behaviour guidelines for children and youth. Appl Physiol Nutr Metab, 36, 59-71.

[36] Tremblay MS, LeBlanc AG, Kho ME, et al. (2011). Systematic review of sedentary behaviour and health indicators in school-aged children and youth. Int J Behav Nutr Phys Act, 8, 98.

[37] Ekelund U, Luan J, Sherar LB, et al. (2012). Moderate to vigorous physical activity and sedentary time and cardiometabolic risk factors in children and adolescents. Jama, 307, 704-712.

[38] Donnelly JE, Hill JO, Jacobsen DJ, et al. (2003). Effects of a 16month randomized controlled exercise trial on body weight and composition in young, overweight men and women: the Midwest Exercise Trial. Arch Intern Med, 163, 1343-1350.

[39] King NA, Hopkins M, Caudwell P, et al. (2008). Individual variability following 12 weeks of supervised exercise: identification and characterization of compensation for exercise-induced weight loss. Int J Obes, 32, 177-184.

[40] Rowland TW. (1998). The biological basis of physical activity. Med Sci Sport. Exerc, 30, 392-399.

[41] Fremeaux AE, Mallam KM, Metcalf BS, et al. (2011). The impact of school-time activity on total physical activity: the activitystat hypothesis (EarlyBird 46). Int J Obes, 35, 1277-1283.

[42] Thivel D \& Duche P. (2014). Physical activity for weight loss in children: is there any compensatory mechanism? Pediatr Exerc Sci, 26, 121-123.

[43] Martinez Vizcaino V, Salcedo Aguilar F, Franquelo Gutierrez R, et al. (2008). Assessment of an after-school physical activity program to prevent obesity among 9- to 10-year-old children: a cluster randomized trial. Int J Obes, 32, 12-22.

[44] Kohl 3rd HW \& Hobbs KE. (1998). Development of physical activity behaviors among children and adolescents. Pediatrics 101 , 549-554.

[45] Lee S, Bacha F, Hannon T, et al. (2012). Effects of aerobic versus resistance exercise without caloric restriction on abdominal fat, intrahepatic lipid, and insulin sensitivity in obese adolescent boys: a randomized, controlled trial. Diabetes, 61, 2787-2795.

[46] Riddoch CJ, Bo Andersen L, Wedderkopp N, et al. (2004). Physical activity levels and patterns of 9- and 15-yr-old European children. Med Sci Sport. Exerc, 36, 86-92. 